\title{
Oscillation and Conversion Performance of Double-Float Wave Energy Converter
}

\author{
Liang Zhang ${ }^{1} \cdot$ Peng Jin ${ }^{1} \cdot$ Binzhen Zhou ${ }^{1} \cdot$ Xiongbo Zheng $^{2} \cdot$ Hengxu Liu ${ }^{1}$
}

Received: 12 October 2017 / Accepted: 6 July 2018 / Published online: 29 March 2019

(C) The Author(s) 2019

\begin{abstract}
In this study, we investigated the hydrodynamic and energy conversion performance of a double-float wave energy converter (WEC) based on the linear theory of water waves. The generator power take-off (PTO) system is modeled as a combination of a linear viscous damping and a linear spring. Using the frequency domain method, the optimal damping coefficient of the generator PTO system is derived to achieve the optimal conversion efficiency (capture width ratio). Based on the potential flow theory and the higher-order boundary element method (HOBEM), we constructed a threedimensional model of double-float WEC to study its hydrodynamic performance and response in the time domain. Only the heave motion of the two-body system is considered and a virtual function is introduced to decouple the motions of the floats. The energy conversion character of the double-float WEC is also evaluated. The investigation is carried out over a wide range of incident wave frequency. By analyzing the effects of the incident wave frequency, we derive the PTO's damping coefficient for the double-float WEC's capture width ratio and the relationships between the capture width ratio and the natural frequencies of the lower and upper floats. In addition, it is capable to modify the natural frequencies of the two floats by changing the stiffness coefficients of the PTO and mooring systems. We found that the natural frequencies of the device can directly influence the peak frequency of the capture width, which may provide an important reference for the design of WECs.
\end{abstract}

Keywords Double-float WEC $\cdot$ Energy conversion $\cdot$ Capture width ratio $\cdot$ Optimal damping $\cdot$ Resonance

\section{Introduction}

Wave energy is an eco-friendly energy that attracts extensive research interest due to its broad global distribution and high energy density (Isaacs and Seymour 1973; Falnes 2007).

\section{Article Highlights}

-The optimal PTO damping is analytically derived for a double-float WEC system.

-Through actively controlled generator damping, a double-float WEC achieves larger capture width ratio.

-Mooring stiffness is used to tune the natural frequency of the doublefloat WEC.

\section{Binzhen Zhou}

zhoubinzhen@hrbeu.edu.cn

1 College of Shipbuilding Engineering, Harbin Engineering University, Harbin 150001, China

2 College of Science, Harbin Engineering University, Harbin 150001, China
Energy conversion in WECs is categorized into oscillating water column (OWC) and point absorber (Falcao 2010) types. The double-float WEC, which is in the point absorber category, has high conversion efficiency and simple construction, and has become a hot spot in the study of wave energy extraction.

In recent years, much work has been performed with respect to energy conversion theories and the hydrodynamics of WECs. Evans (1976) introduced the first efficiency equation of a one-body WEC. Two-body WECs are also studied in various literature $(\mathrm{Yu}$ and $\mathrm{Li}$ 2013; Kim et al. 2016; Son et al. 2016; Dai et al. 2017; Son and Yeung 2017; Cho and Kim 2017; Liang and Zuo 2017). Falnes (1999) used the frequency domain method to treat a two-body WEC as a one-body WEC. The maximum efficiency can be achieved with an optimal damping coefficient and optimal stiffness coefficient. Eriksson et al. (2005) studied the damped oscillation and efficiency of a cylindrical WEC in both regular waves and real marine conditions. Wu et al. (2013) studied the radiation and diffraction problems of both floating-cylinder 
and fixed-cylinder WECs using an analytical approach and determined the relationships between efficiency, the stiffness coefficient, and the geometry of a WEC, although the relationship between efficiency and the natural frequencies of floats were not clearly explained. Zhang et al. (2016) used an analytical method to study the relationship between the natural frequencies of floats and the conversion efficiency of the WEC, but focused on the effects of the damping plate, PTO stiffness coefficient, and mooring stiffness coefficient. Neither $\mathrm{Wu}$ et al. (2013) nor Zhang et al. (2016) proposed an expression for the optimal PTO damping coefficient.

In this paper, we first derive the power absorption and conversion efficiency of the double-float WEC. Considering the PTO damping coefficient, stiffness coefficient, and mooring stiffness, we derive the optimal damping coefficient of the double-float WEC using the frequency domain method. Based on the potential flow theory, we apply the higher-order boundary element method (HOBEM) to construct a 3D model of the double-float WEC in the linear time domain. To decouple the motion of the floats, we introduce a virtual function. We then validate the proposed model by comparing it with the optimal damping coefficient derived from frequency domain method. We investigate the influence of incident wave frequency, PTO damping coefficient, stiffness coefficient, and mooring stiffness coefficient on the motion response of the floats and the conversion efficiency.

\section{Mathematical Model}

The double-float WEC includes upper and lower floats (Fig. 1) connected by a flexible string and the rotor of the linear permanent magnet generator. The upper float is a circular ring with an outer radius $R_{1}$ and draught $H_{1}$. The central lower float, which has a radius $R_{2}$, is equipped with a generator coil and a cylindrical damping plate with a radius $R_{3}$ and height $H_{3}$ attached underneath. The damping plate is moored to the seabed. The distance from the bottom of the upper float and the top of the damping plate is $H_{2}$. The water depth is $d$. When excited by waves, the relative motion between the two floats generates electric power. The two floats are connected by a sping with an elasity coefficient of $K_{\text {pto }}$. The magnet force from the generator provides a damping effect with a damping coefficient of $B_{\mathrm{pto}}$. Wave power is absorbed by this PTO system. We use the Cartesian coordinate system OXYZ. The Zaxis rises vertically upward and coincides with the central line of the WEC. The $X$ - and $Y$-axes are on the calm water surface.

To describe the motion of the WEC in this paper, we assume a linear wave motion, and assume that the fluid is inviscid, incompressible, and irrotational. This simplifies the effect

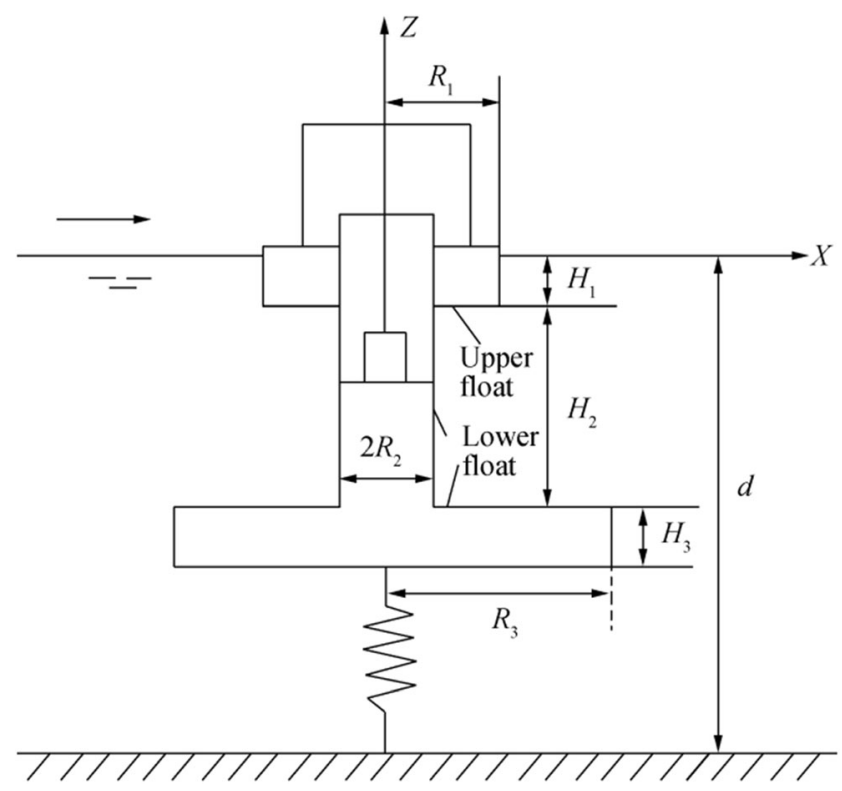

Fig. 1 Schematic of two-body wave energy device

of the PTO system as a linear damper and a spring, and also simplifies the mooring line as a linear spring $K_{\mathrm{m}}$. Figure 2 shows a diagram of the floats. As the absorption of wave energy mainly depends on the relative heave motion of the floats, the motion in the other five degrees of freedom is not significant and is not considered. The motion functions of the two floats are as follows:

$m_{1} \ddot{z}_{1}+B_{\text {pto }}\left(z_{1}-z_{2}\right)+K_{\text {pto }}\left(z_{1}-z_{2}\right)=f_{1}$

$m_{2} \ddot{z}_{2}+B_{\text {pto }}\left(z_{2}-z_{1}\right)+K_{\text {pto }}\left(z_{2}-z_{1}\right)+K_{\mathrm{m}} z_{2}=f_{2}$

where $m_{1}$ and $m_{2}$ are the mass, and $z_{1}$ and $z_{2}$ are the vertical displacements of the floats, $z_{1}$ and $z_{2}$ are their velocities in the vertical direction, and $\ddot{z}_{1}$ and $\ddot{z}_{2}$ are their accelerations. $f_{1}$ and $f_{2}$ are the body forces on the floats.

\section{Energy Conversion Efficiency}

The heave displacement of the float is written as follows:

$z_{j}=\operatorname{Re}\left(Z_{j} \mathrm{e}^{-\mathrm{i} \omega t}\right)$

where $j=1$ for the upper float, $j=2$ for the lower float, $\omega$ is the incident wave frequency, and $Z_{1}$ and $Z_{2}$ are the complex amplitudes of the upper and lower floats with respect to the heave motion, respectively.

The energy absorption rate of the WEC in a unit of time is defined as the work done by the fluid in the WEC. The average power absorption efficiency of the WEC (Evans 1976) is as follows: 


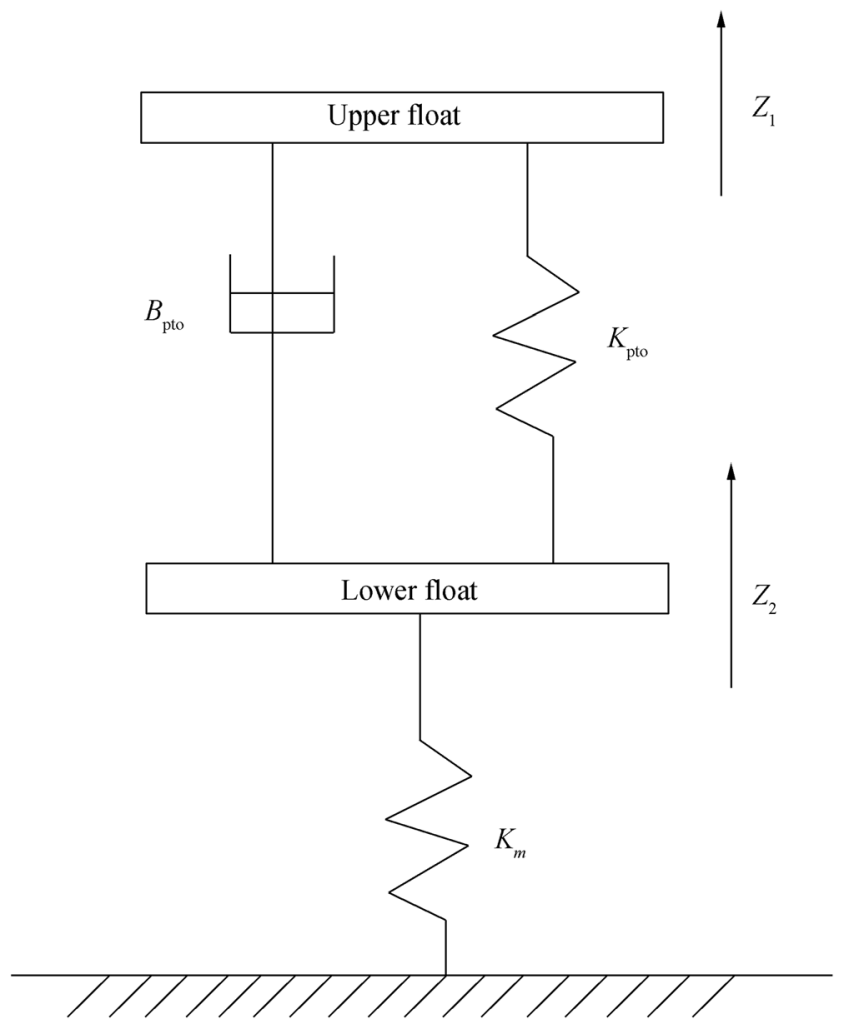

Fig. 2 Force diagram of two-body wave energy device

$E_{\text {pto }}=\frac{1}{2} B_{\text {pto }} \omega^{2}\left|Z_{1}-Z_{2}\right|^{2}$

The capture width ratio of a WEC is defined as the ratio of the wave energy captured by the WEC to the total input wave energy within the width of the WEC, as follows:
$\beta=\frac{E_{\mathrm{pto}}}{D \cdot E_{w}}$

where $E_{w}$ is the incident wave power per unit width, i.e.,

$E_{w}=\frac{1}{16} \rho g A^{2} \frac{\omega}{k_{0}}\left(1+\frac{2 k_{0} d}{\sinh 2 k_{0} d}\right)$

where $D$ is the diameter of the float, $E_{w}$ is the incident wave power per unit width (Basco 1984), $\rho$ is the water density, $A$ is the incident wave amplitude, and $k_{0}$ is the wave number.

\section{Optimal PTO Damping}

Based on frequency domain theory, Eqs. (1) and (2) can be written as follows:

$$
\begin{aligned}
& \left(m_{1}+\mu_{11}\right) \ddot{z}_{1}+\mu_{12} \ddot{z}_{2}+B_{\text {pto }}\left(z_{1}-\dot{z}_{2}\right)+\lambda_{11} \dot{z}_{1} \\
& +\lambda_{12} z_{2}+K_{\text {pto }}\left(z_{1}-z_{2}\right)+C_{1} z_{1}=F_{d 1} \\
& \left(m_{2}+\mu_{22}\right) \ddot{z}_{2}+\mu_{21} \ddot{z}_{1}+B_{\text {pto }}\left(z_{2}-z_{1}\right)+\lambda_{22} z_{2} \\
& +\lambda_{21} z_{1}+K_{\text {pto }}\left(z_{2}-z_{1}\right)+C_{2} z_{2}+K_{m} z_{2}=F_{d 2}
\end{aligned}
$$

where $F_{d 1}$ and $F_{d 2}$ are the excitation forces and $C_{1}$ and $C_{2}$ are the restoration forces on the upper and lower floats, respectively. $\mu_{i j}$ and $\lambda_{i j}$ are the added mass and radiation damping coefficients, respectively, exerted on the $i$ th float by the $j$ th float. We calculated these coefficients using HydroStar software. Let $F_{d 1}=\operatorname{Re}\left(f_{d 1} \mathrm{e}^{-\mathrm{i} \omega t}\right)$ and $F_{d 2}=\operatorname{Re}\left(f_{d 2} \mathrm{e}^{-\mathrm{i} \omega t}\right)$, where $f_{d 1}$ and $f_{d 2}$ are the complex amplitudes of the upper and lower floats, respectively. By substituting them with Eq. (3) into Eqs. (11) and (12), we have the following:

$$
\left[\begin{array}{cc}
-\omega^{2}\left(m_{1}+\mu_{11}\right)-\mathrm{i} \omega\left(B_{\text {pto }}+\lambda_{11}\right)+K_{\text {pto }}+C_{1} & -\omega^{2} \mu_{12}+\mathrm{i} \omega\left(B_{\text {pto }}-\lambda_{12}\right)-K_{\text {pto }} \\
-\omega^{2} \mu_{21}+\mathrm{i} \omega\left(B_{\text {pto }}-\lambda_{21}\right)-K_{\text {pto }} & -\omega^{2}\left(m_{2}+\mu_{22}\right)-\mathrm{i} \omega\left(B_{\text {pto }}+\lambda_{22}\right)+K_{\text {pto }}+C_{2}+K_{m}
\end{array}\right]\left[\begin{array}{l}
Z_{1} \\
Z_{2}
\end{array}\right]=\left[\begin{array}{l}
f_{d 1} \\
f_{d 2}
\end{array}\right]
$$

Let

$$
\begin{aligned}
& Y_{1}=Y_{r 1}+\mathrm{i} Y_{i 1}=-\omega^{2}\left(m_{1}+\mu_{11}\right)+C_{1}-\mathrm{i} \omega \lambda_{11} \\
& Y_{2}=Y_{2 r}+\mathrm{i} Y_{2 i}=-\omega^{2}\left(m_{2}+\mu_{22}\right)+C_{2}+K_{m}-\mathrm{i} \omega \lambda_{22} \\
& Y_{12}=Y_{12 r}+\mathrm{i} Y_{12 i}=-\omega^{2} \mu_{12}-\mathrm{i} \omega \lambda_{12} \\
& Y_{21}=Y_{21 r}+\mathrm{i} Y_{21 i}=-\omega^{2} \mu_{21}-\mathrm{i} \omega \lambda_{21}
\end{aligned}
$$

where $Y_{1}$ is the hydrodynamic coefficient associated with the self-motion of the upper float, $Y_{2}$ is the hydrodynamic coefficient associated with the self-motion of the lower float, $Y_{12}$ is the hydrodynamic coefficient of the upper float caused by the lower float, and $Y_{21}$ is the hydrodynamic coefficient of the lower float caused by the upper float. Equation (9) can be rewritten as follows:

$$
\left[\begin{array}{cc}
Y_{1}-\mathrm{i} \omega B_{\mathrm{pto}}+K_{\mathrm{pto}} & Y_{12}+\mathrm{i} \omega B_{\mathrm{pto}}-K_{\mathrm{pto}} \\
Y_{21}+\mathrm{i} \omega B_{\mathrm{pto}}-K_{\mathrm{pto}} & Y_{2}-\mathrm{i} \omega B_{\mathrm{pto}}+K_{\mathrm{pto}}
\end{array}\right]\left[\begin{array}{l}
Z_{1} \\
Z_{2}
\end{array}\right]=\left[\begin{array}{l}
f_{d 1} \\
f_{d 2}
\end{array}\right]
$$

Let

$$
A=\left[\begin{array}{cc}
Y_{1}-\mathrm{i} \omega B_{\text {pto }}+K_{\text {pto }} & Y_{12}+\mathrm{i} \omega B_{\text {pto }}-K_{\text {pto }} \\
Y_{21}+\mathrm{i} \omega B_{\text {pto }}-K_{\text {pto }} & Y_{2}-\mathrm{i} \omega B_{\text {pto }}+K_{\text {pto }}
\end{array}\right]
$$


By solving the equations, we have the following:

$\left[\begin{array}{l}Z_{1} \\ Z_{2}\end{array}\right]=\frac{1}{|A|}\left[\begin{array}{l}f_{d 1}\left(Y_{2}-\mathrm{i} \omega B_{\text {pto }}+K_{\text {pto }}\right)-f_{d 2}\left(Y_{12}+\mathrm{i} \omega B_{\text {pto }}-K_{\text {pto }}\right) \\ f_{d 2}\left(Y_{1}-\mathrm{i} \omega B_{\text {pto }}+K_{\text {pto }}\right)-f_{d 1}\left(Y_{21}+\mathrm{i} \omega B_{\text {pto }}-K_{\text {pto }}\right)\end{array}\right]$

Thus:

$Z_{1}-Z_{2}=\frac{1}{|A|}\left[f_{d 1}\left(Y_{2}+Y_{21}\right)-f_{d 2}\left(Y_{12}+Y_{1}\right)\right]$
By representing $Y_{1}, Y_{2}, Y_{12}$, and $Y_{21}$ with complex numbers and substituting them into $A$, we have:

$$
A=\left[\begin{array}{cc}
\left(Y_{1 r}+K_{\mathrm{pto}}\right)-\mathrm{i}\left(\omega B_{\mathrm{pto}}-Y_{1 i}\right) & \left(Y_{12 r}-K_{\mathrm{pto}}\right)+\mathrm{i}\left(Y_{12 i}+\omega B_{\mathrm{pto}}\right) \\
\left(Y_{21 r}-K_{\mathrm{pto}}\right)+\mathrm{i}\left(Y_{21 i}+\omega B_{\mathrm{pto}}\right) & \left(Y_{2 r}+K_{\mathrm{pto}}\right)-\mathrm{i}\left(\omega B_{\mathrm{pto}}-Y_{2 i}\right)
\end{array}\right]
$$

The determinant of $A$ is as follows:

$|A|=\left[\left(\left(Y_{1 r}+K_{\text {pto }}\right)\left(Y_{2 r}+K_{\text {pto }}\right)-\left(Y_{12 r}-K_{\text {pto }}\right)\left(Y_{21 r}-K_{\text {pto }}\right)-Y_{1 i} Y_{2 i}+Y_{12 i} Y_{21 i}\right)+\omega\left(Y_{1 i}+Y_{2 i}+Y_{12 i}+Y_{21 i}\right) B_{\text {pto }}\right]-$
$i\left[\left(Y_{12 i}\left(Y_{21 r}-K_{\text {pto }}\right)-Y_{2 i}\left(Y_{1 r}+K_{\text {pto }}\right)-Y_{1 i}\left(Y_{2 r}+K_{\text {pto }}\right)+Y_{21 i}\left(Y_{12 r}-K_{\text {pto }}\right)\right)+\omega\left(Y_{12 r}+Y_{21 r}+Y_{1 r}+Y_{2 r}\right) B_{\text {pto }}\right]$

By substituting Eqs. (17) and (19) into Eq. (4), we have

$$
\begin{aligned}
& E_{\text {pto }}=\frac{1}{2} B_{\text {pto }} \omega^{2} \frac{\left|f_{d 1}\left(Y_{2}+Y_{21}\right)-f_{d 2}\left(Y_{12}+Y_{1}\right)\right|^{2}}{N} \\
& N=\left[\left(\left(Y_{1 r}+K_{\text {pto }}\right)\left(Y_{2 r}+K_{\text {pto }}\right)-\left(Y_{12 r}-K_{\text {pto }}\right)\left(Y_{21 r}-K_{\text {pto }}\right)-Y_{1 i} Y_{2 i}+Y_{12 i} Y_{21 i}\right)+\omega\left(Y_{1 i}+Y_{2 i}+Y_{12 i}+Y_{21 i}\right) B_{\text {pto }}\right]^{2}+ \\
& {\left[\left(Y_{12 i}\left(Y_{21 r}-K_{\text {pto }}\right)-Y_{2 i}\left(Y_{1 r}+K_{\text {pto }}\right)-Y_{1 i}\left(Y_{2 r}+K_{\text {pto }}\right)+Y_{21 i}\left(Y_{12 r}-K_{\text {pto }}\right)\right)+\omega\left(Y_{12 r}+Y_{21 r}+Y_{1 r}+Y_{2 r}\right) B_{\text {pto }}\right]^{2}}
\end{aligned}
$$

where $N$ is the square of the determinant of $A$.
Let

$$
\begin{aligned}
M= & \frac{1}{2} \omega^{2}\left|f_{d 1}\left(Y_{2}+Y_{21}\right)-f_{d 2}\left(Y_{12}+Y_{1}\right)\right|^{2} \\
O= & \left(Y_{1 r}+K_{\mathrm{pto}}\right)\left(Y_{2 r}+K_{\mathrm{pto}}\right)-\left(Y_{12 r}-K_{\mathrm{pto}}\right)\left(Y_{21 r}-K_{\mathrm{pto}}\right) \\
& -Y_{1 i} Y_{2 i}+Y_{12 i} Y_{21 i}
\end{aligned}
$$$$
P=\omega\left(Y_{1 i}+Y_{2 i}+Y_{12 i}+Y_{21 i}\right)
$$$$
Q=Y_{12 i}\left(Y_{21 r}-K_{\text {pto }}\right)-Y_{2 i}\left(Y_{1 r}+K_{\text {pto }}\right)
$$$$
-Y_{1 i}\left(Y_{2 r}+K_{\text {pto }}\right)+Y_{21 i}\left(Y_{12 r}-K_{\text {pto }}\right)
$$$$
R=\omega\left(Y_{12 r}+Y_{21 r}+Y_{1 r}+Y_{2 r}\right) \text {. }
$$

Then, we have

$$
E_{\mathrm{pto}}=\frac{M}{\left(O^{2}+Q^{2}\right) \frac{1}{B_{\mathrm{pto}}}+\left(P^{2}+R^{2}\right) B_{\mathrm{pto}}+2(O P+Q R)}
$$

When:

$$
B_{\text {pto }}=\sqrt{\frac{O^{2}+Q^{2}}{P^{2}+R^{2}}}
$$

$E_{\text {pto }}$ reaches its maximum value:

$$
E_{\text {pto }}=\frac{M}{2 \sqrt{\left(O^{2}+Q^{2}\right)\left(P^{2}+R^{2}\right)}+2(O P+Q R)},
$$

and this damping coefficient is denoted as $B_{\text {opt }}$.

\section{Time Domain Model}

The wave-structure interaction model in open water is established using the incident wave and diffraction separation method based on potential flow theory (Zhou et al. 2015; Zhou and $\mathrm{Wu} 2015)$. The diffraction potential satisfies the Laplace equation and the following boundary conditions: 
The boundary conditions on the free surface are:

$\frac{\partial \eta_{a}}{\partial t}=\frac{\partial \phi_{a}}{\partial z}-\delta(r) \eta_{a}$

$\frac{\partial \phi_{a}}{\partial t}=-g \eta_{a}-\delta(r) \phi_{a}$

$\frac{\partial \phi_{a}}{\partial \boldsymbol{n}}=\boldsymbol{V} \cdot \boldsymbol{n}-\frac{\partial \phi_{b}}{\partial \boldsymbol{n}}$

where $\phi_{\mathrm{b}}$ and $\eta_{\mathrm{b}}$ are the incident wave potential and wave height, respectively. According to the linear wave theory, $\phi_{\mathrm{a}}$ and $\eta_{\mathrm{a}}$ are the diffraction potential and diffraction wave height, respectively; and $\delta(r)$ is a function for absorbing a diffraction wave on the outer boundary. $\boldsymbol{V}$ is the velocity of the body, and $\boldsymbol{n}$ is the normal vector pointing outside the body surface.

Based on the time domain theory, the motion equations of the floats are as follows:

$m_{1} \ddot{z}_{1}+B_{\text {pto }}\left(z_{1}-z_{2}\right)+K_{\text {pto }}\left(z_{1}-z_{2}\right)=f_{1}^{\prime}+f_{g 1}$

$m_{2} \ddot{z}_{2}+B_{\text {pto }}\left(z_{2}-z_{1}\right)+K_{\text {pto }}\left(z_{2}-z_{1}\right)+K_{m} z_{2}=f_{2}^{\prime}+f_{g 2}$

where $f_{1}^{\prime}$ and $f_{2}^{\prime}$ are the forces exerted on the above-water and lower floats, which can be derived using a pressure integral on the average wet surfaces $S_{B 1}$ and $S_{B 2}$ :

$f_{1}^{\prime}=\iint_{S_{B 1}} p n_{3} \mathrm{~d} s=-\rho \iint_{S_{B 1}}\left(\phi_{t}+g z\right) n_{3} \mathrm{~d} s$

$f_{2}^{\prime}=\iint_{S_{B 2}} p n_{3} \mathrm{~d} s=-\rho \iint_{S_{B 2}}\left(\phi_{t}+g z\right) n_{3} \mathrm{~d} s$

When using the acceleration potential method (Tanizawa 1995) to calculate $\phi_{t}$ in Eqs. (35) and (36), the acceleration of the body at that constant is needed. At the same time, the acceleration of the body must be calculated by the motion equations, which require a known $\phi_{t}$. The calculation of $\phi_{t}$ and the acceleration of the body is therefore a process of iterative inductions. On the other hand, the problem in this study involves two floats, which means that two unknown accelerations are in the equation. Zhou et al. (2016) proposed a virtual function method to solve these two problems.

We introduce the virtual function $\psi_{3}$ that satisfies the Laplace equation. By Green's second identity $\psi_{3}$ satisfies the following:

$\iint_{S_{B 1}+S_{B 2}+S_{F}}\left(\left(\phi_{a}\right)_{t} \frac{\partial \psi_{3}}{\partial n}-\psi_{3} \frac{\partial\left(\phi_{a}\right)_{t}}{\partial n}\right) \mathrm{d} s=0$

When calculating the forces on the upper float, let the boundary condition of $\psi_{3}$ be the following: $\begin{cases}\psi_{3}=0 & \text { on } S_{F} \\ \frac{\partial \psi_{3}}{\partial n}=n_{3} & \text { on } S_{B 1} \\ \frac{\partial \psi_{3}}{\partial n}=0 & \text { on } S_{B 2}\end{cases}$

When calculating the forces on the lower float, let the boundary condition of $\psi_{3}$ be as follows:

$\begin{cases}\psi_{3}=0 & \text { on } S_{F} \\ \frac{\partial \psi_{3}}{\partial n}=0 & \text { on } S_{B 1} \\ \frac{\partial \psi_{3}}{\partial n}=n_{3} & \text { on } S_{B 2}\end{cases}$

By substituting the virtual function boundary conditions of Eqs. (42) and (43) into Eq. (41) and calculating the pressure integral Eqs. (39) and (40), we obtain Eq. (44) for the two accelerations $\ddot{z}_{1}$ and $\ddot{z}_{2}$. Then, we can decouple the abovewater and lower floats by solving the equation. After the accelerations are computed, we use the 4th-order Runge-Kutta method to update the velocity and displacement at the next constant.

$\left\{\begin{array}{l}A_{1} \ddot{z}_{1}=F_{1}+A_{12} \ddot{z}_{2} \\ A_{2} \ddot{z}_{2}=F_{2}+A_{21} \ddot{z}_{1}\end{array}\right.$

where:

$A_{1}=m_{1}+\rho \iint_{S_{B 1}} \psi_{3} n_{3} \mathrm{~d} s$

$A_{2}=m_{2}+\rho \iint_{S_{B 2}} \psi_{3} n_{3} \mathrm{~d} s$

$A_{12}=-\rho \iint_{S_{B 2}} \psi_{3} n_{3} \mathrm{~d} s$

$A_{21}=-\rho \iint_{S_{B 1}} \psi_{3} n_{3} \mathrm{~d} s$

$F_{1}=-\rho \iint_{S_{B 1}}\left(g z+\frac{\partial \phi_{\mathrm{b}}}{\partial t}\right) \frac{\partial \psi_{3}}{\partial n} \mathrm{~d} s$

$-\rho \iint_{S_{F}}\left(g\left(\eta_{a}+\eta_{b}\right)+\frac{\partial \phi_{\mathrm{b}}}{\partial t}\right) \frac{\partial \psi_{3}}{\partial n} \mathrm{~d} s$

$+\rho \iint_{S_{B 1}+S_{B 2}} \psi_{3} \frac{\partial\left(\phi_{\mathrm{b}}\right)_{t}}{\partial n} \mathrm{~d} s$

$+f_{g 1}-B_{\text {pto }}\left(z_{1}-z_{2}\right)-K_{\text {pto }}\left(z_{1}-z_{2}\right)$ 


$$
\begin{aligned}
& F_{2}=-\rho \iint_{S_{B 2}}\left(g z+\frac{\partial \phi_{\mathrm{b}}}{\partial t}\right) \frac{\partial \psi_{3}}{\partial n} \mathrm{~d} s \\
& -\rho \iint_{S_{F}}\left(g\left(\eta_{a}+\eta_{b}\right)+\frac{\partial \phi_{b}}{\partial t}\right) \frac{\partial \psi_{3}}{\partial n} \mathrm{~d} s \\
& \quad+\rho \iint_{S_{B 1}+S_{B 2}} \psi_{3} \frac{\partial\left(\phi_{b}\right)_{t}}{\partial n} \mathrm{~d} s \\
& +f_{g 2}-B_{\text {pto }}\left(z_{2}-z_{1}\right)-K_{\mathrm{pto}}\left(z_{2}-z_{1}\right)-K_{m} z_{2}
\end{aligned}
$$

\section{Numerical Results}

\subsection{Validation}

To verify the accuracy of our proposed numerical model, we performed simulations of the double-float WEC with different PTO damping coefficients in linear waves. Table 1 lists the main parameters of the WEC. Figure 3 shows the relationship between the absorption power $E_{\text {pto }}$ and the PTO damping coefficient $B_{\text {pto }}$ with the incident wave period $T=7 \mathrm{~s}$, PTO stiffness coefficient $K_{\text {pto }}=2 \times$ $10^{4} \mathrm{~N} / \mathrm{m}$, and mooring stiffness coefficient $K_{\mathrm{m}}=0$. As $B_{\text {pto }}$ increases, the absorption power of the WEC first increases and then decreases. When $B_{\text {pto }}=1.92 \times 10^{6} \mathrm{~N}$. $\mathrm{s} / \mathrm{m}$, the absorption power reaches its peak value. This result agrees well with the optimal PTO damping coefficient $B_{\text {opt }}=1.90 \times 10^{6} \mathrm{~N} \mathrm{~s} / \mathrm{m}$ calculated by Eq. (32), based on the frequency domain theory. Therefore, our proposed model is validated.

\subsection{Effect of $B_{\text {pto }}$ on Conversion Efficiency}

In this section, we analyze the effect of $B_{\mathrm{pto}}$ on the conversion efficiency of the WEC. Here, we denote $B_{\text {opt }}$ as the optimal PTO damping coefficient that makes the absorption power

Table 1 Main parameters of device

\begin{tabular}{ll}
\hline Variables & Value \\
\hline$R_{1} / \mathrm{m}$ & 5.5 \\
$R_{2} / \mathrm{m}$ & 0.592 \\
$R_{3} / \mathrm{m}$ & 7.0 \\
$d / \mathrm{m}$ & 70.0 \\
$H_{1} / \mathrm{m}$ & 0.9 \\
$H_{2} / \mathrm{m}$ & 32.5 \\
$H_{3} / \mathrm{m}$ & 0.84 \\
$m_{1} / \mathrm{kg}$ & 86.5 \\
$m_{2} / \mathrm{kg}$ & 169.9 \\
\hline
\end{tabular}

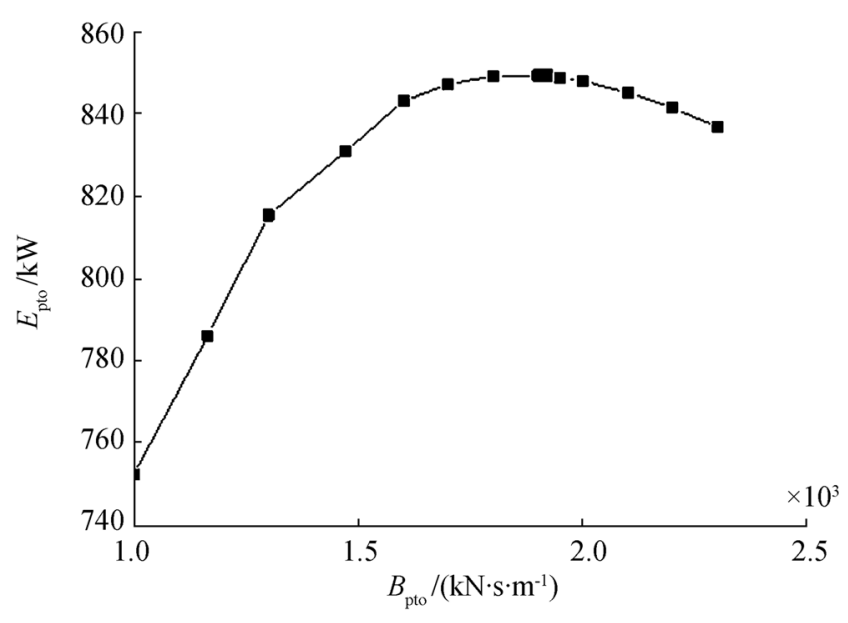

Fig. 3 Absorption power for different PTO damping coefficients, at $T=$ $7 \mathrm{~s}, K_{\text {pto }}=2 \times 10^{4} \mathrm{~N} / \mathrm{m}$, and $K_{m}=0$

reach its peak value. We study the influence of the incident wave frequency on the capture width ratio and motion

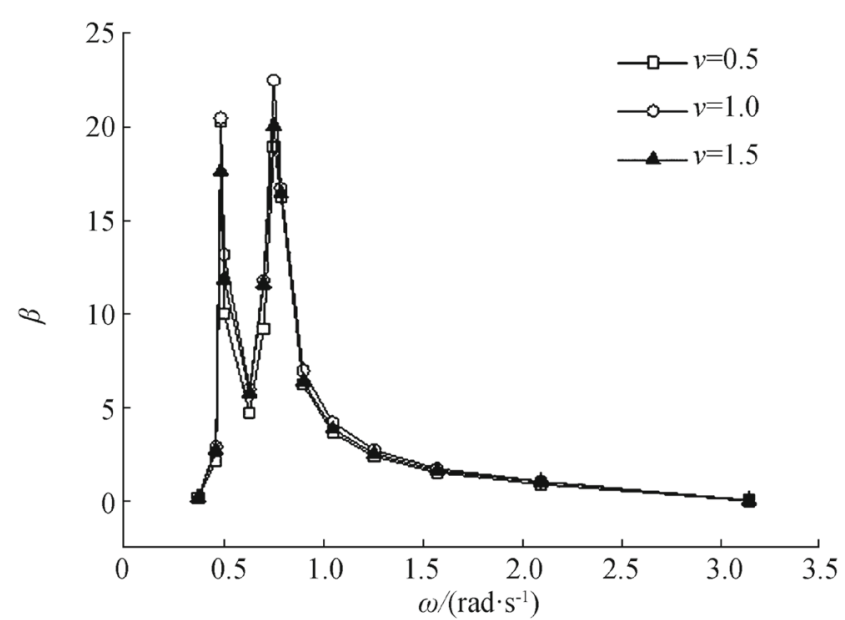

(a) Capture width ratio of the WEC

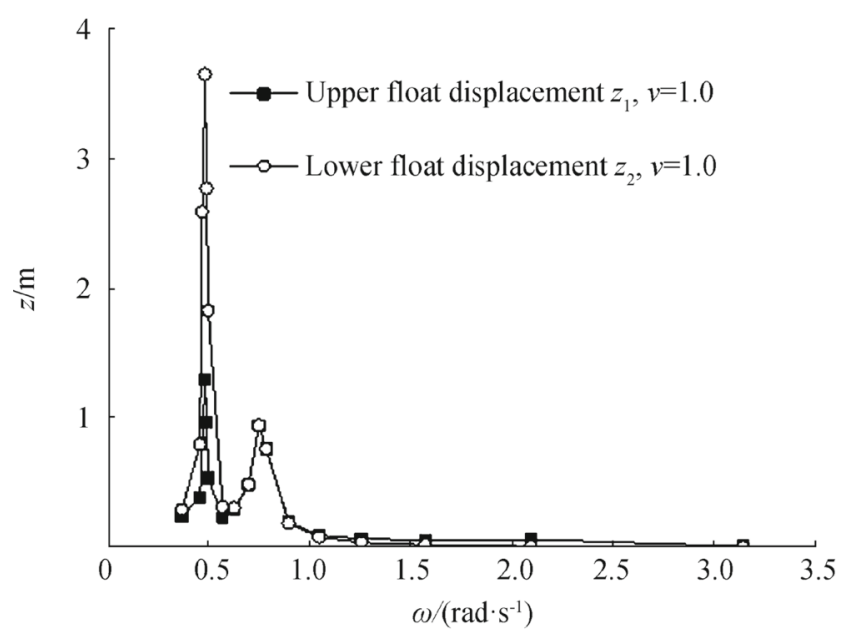

(b) Motion response of the two floats

Fig. 4 Capture width ratios and motion responses for different values of $B_{\text {pto }}$ and $\nu$ 


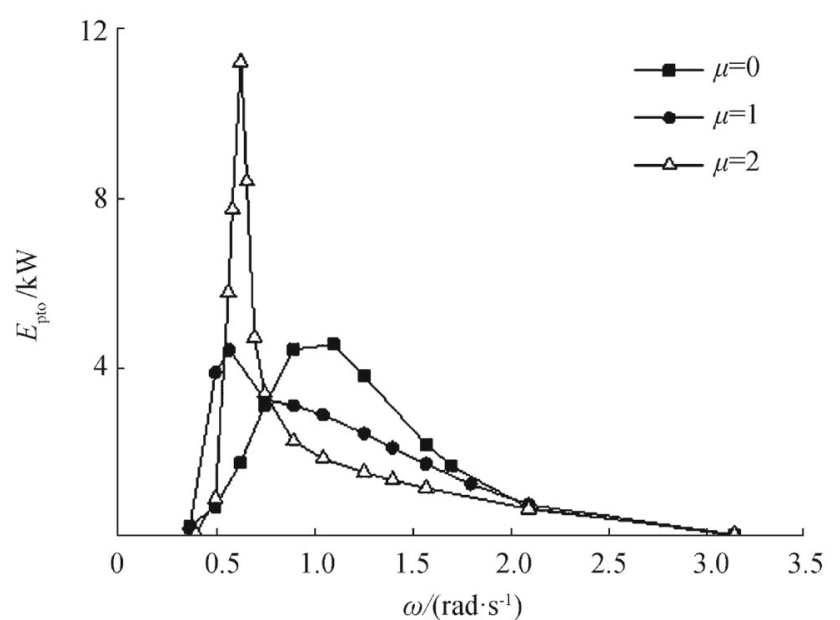

(a) Capture width ratio

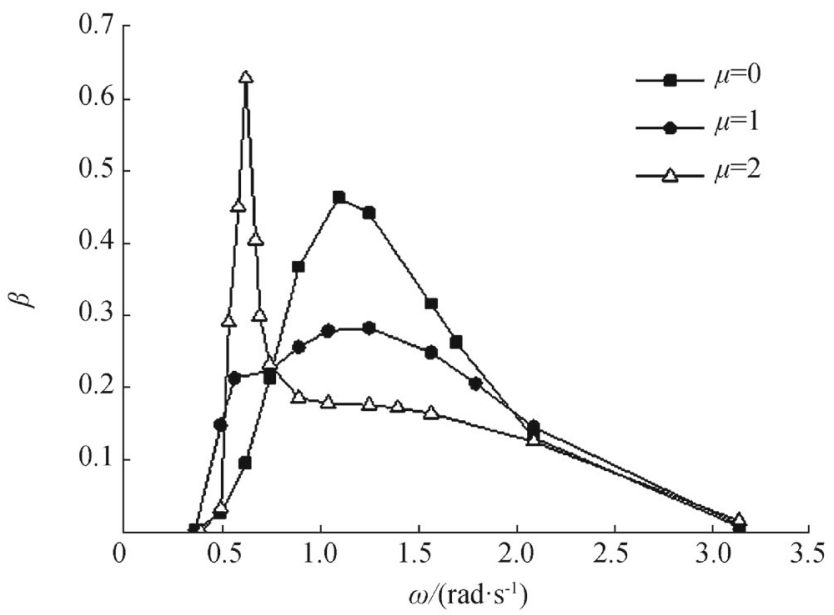

(b) Absorption powers

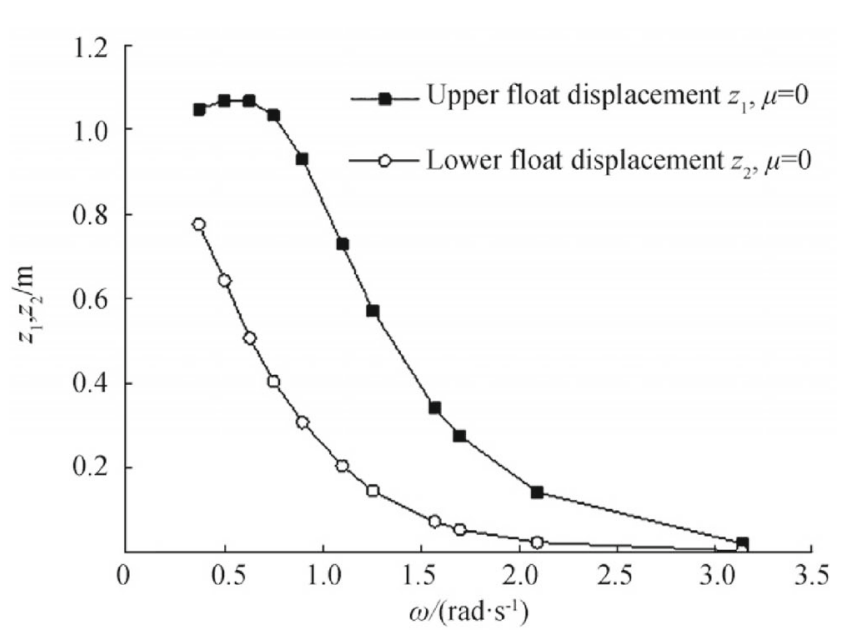

(c) Motion response for $\boldsymbol{\mu}=0$

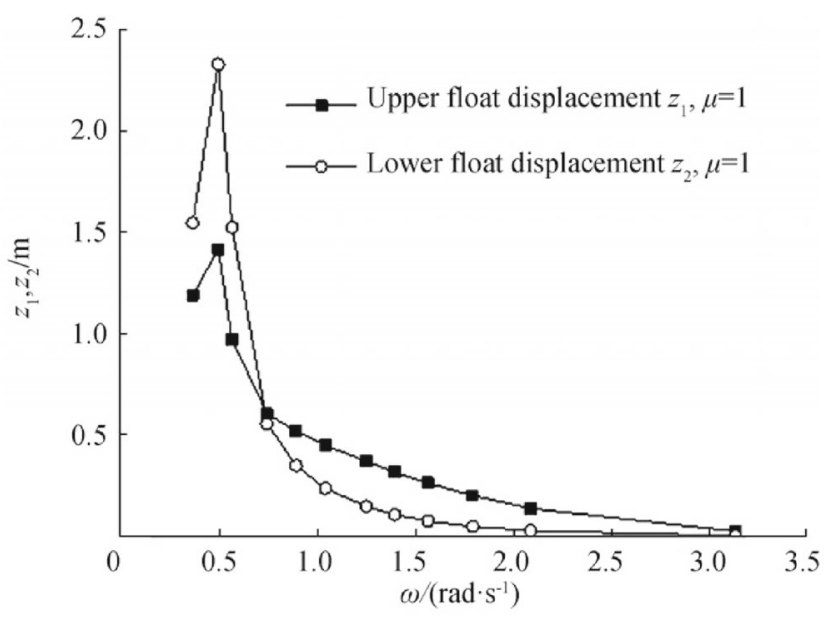

(d) Motion response for $\mu=1$

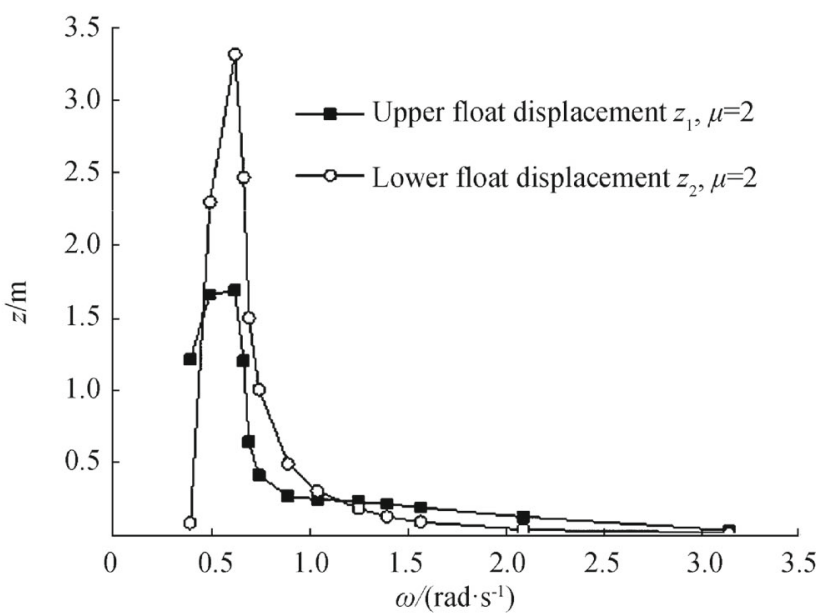

(e) Motion response for $\mu=2$

Fig. 5 Capture width ratios, absorption powers, and motion responses for different values of $B_{\text {pto }}$ and $\nu$

response of the floats at $v=B_{\text {pto }} / B_{\text {opt }}$ equal to $0.5,1$, and 1.5 with $K_{\text {pto }}=4 \times 10^{5} \mathrm{~N} / \mathrm{m}$ and $K_{m}=0$ (Fig. 4). In Fig. 4a, the capture width ratio at $B_{\text {pto }} / B_{\text {opt }}=1$ is larger than that at $B_{\text {pto }} /$
$B_{\text {opt }}=0.5$ or 1.5 . The difference is even greater for the peak value. On the other hand, the capture width ratio has two peak values with incident frequencies of $\omega=0.48$ and 0.75 . 
Figure $4 \mathrm{~b}$ shows the relationship between the motion responses of the floats and the incident wave frequencies. When $\omega=0.48$, the displacement of the lower float is much larger than that of the upper float; when $\omega=0.75$, the displacements of the two floats are similar; when $\omega$ is far away from the peak frequency, the displacement of the upper float is much larger than that of the lower float. The displacements of the floats at $\omega=0.48$ are much larger than those at $\omega=0.75$. One reason for this is that $B_{\mathrm{opt}}$ at $\omega=0.75$ is much larger than $B_{\text {opt }}$ at $\omega=0.48$, and the relative motion between the two floats is small. The other reason is that the lower float is resonant at $\omega=0.48$, whereas the upper float is resonant at $\omega=0.75$. The restoration force of the upper float is much larger than that of the lower float, which means that the motion of the lower float is large and the motion of the upper float is small. When $\omega=0.48$, the resonant lower float has a small restoration force coefficient; therefore, it oscillates with large amplitude. The upper float with a large restoration coefficient will not move identically with the lower float. When $\omega=0.75$, the restoration force coefficient of the lower float is small, and it will move with the upper float.

From the above analysis, we find that the two peaks on the conversion efficiency curve are caused by the respective resonances of the two floats. Next, we analyze the influence of the resonant frequencies of the floats on the capture width ratio of the WEC. Here, the coupled effects are not considered. The natural frequencies of the two floats are derived from the un-damped linear equation for free motion in the frequency domain, as follows:

$$
\omega_{\mathrm{n}}{ }^{1}=\sqrt{\frac{K_{\mathrm{pto}}+C_{1}}{m_{1}+\mu_{11}}} \quad \omega_{n}^{2}=\sqrt{\frac{K_{\mathrm{pto}}+C_{2}+K_{m}}{m_{2}+\mu_{22}}}
$$

where $\mu_{11}$ and $\mu_{12}$ are the added masses of the two floats as they move in the heave direction, which we calculated using HydroStar.

When $K_{\text {pto }}=4 \times 10^{5} \mathrm{~N} / \mathrm{m}$, the natural frequency of the upper float is 2.07 and that of the underwater float is 0.58 . The natural frequency of the lower float is near the first peak frequency of 0.48 in Fig. 4a, but the natural frequency of the upper float is much larger than the 0.75 value. This is because $K_{\text {pto }}$ is a coupled term associated with the relative motion between the two floats, which can be seen from Eq. (47). If the coupled effect is neglected, the numerator in Eq. (47) is larger than its exact value. Therefore, the calculated natural frequency is larger than the peak frequency in Fig. 4. On the other hand, the mass and added mass of the upper float in the denominator is much smaller than those of the lower float, so the influence of the coupled effects is much greater. From Eq. (47), we find that the natural frequency is related to the mass, added mass, restoration coefficient, PTO stiffness coefficient, and mooring stiffness coefficient. Zhang et al. (2016) studied the influence of the geometry and mass of the WEC on conversion efficiency. In the following, we vary the PTO stiffness coefficient $K_{\text {pto }}$ and mooring stiffness coefficient $K_{m}$ to modify the natural frequencies of the floats to investigate their influence on the conversion efficiency of the WEC.

\subsection{Effect of $K_{\text {pto }}$ on Conversion Efficiency}

In this section, we analyze the effect of $K_{\text {pto }}$ on the conversion efficiency of the WEC. If we let $\varepsilon=K_{\text {pto }} /\left(4 \times 10^{5} \mathrm{~N} /\right.$ $\mathrm{m})$, when $B_{\text {pto }}=4 \times 10^{5} \mathrm{~N} \cdot \mathrm{s} / \mathrm{m}$ and $K_{m}=0$, and consider $\varepsilon=0,1$, and 2 , in Fig. 5 , it shows the corresponding absorption powers, conversion efficiencies, and motion responses of the two floats. When $\varepsilon=1$ or 2 , a sharp peak appears in the low-frequency regime (Fig. 5a). When $\varepsilon=0$, a gentle peak appears in the high-frequency regime (Fig. 5c). In Fig. 5c, the displacements of the upper and lower floats have no peak but their relative displacements are large. The reason for this is that when $\varepsilon=0$, no apparent

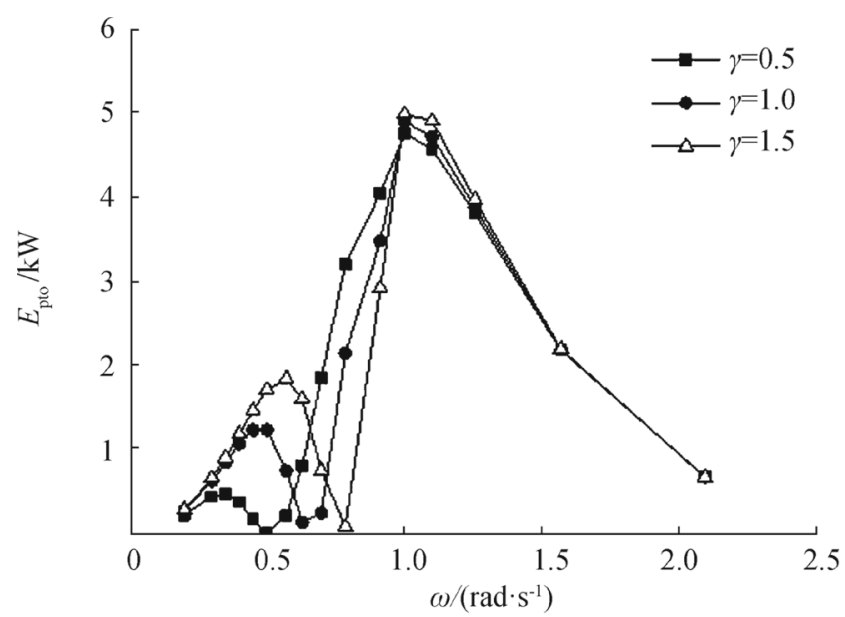

(a) Capture width ratios

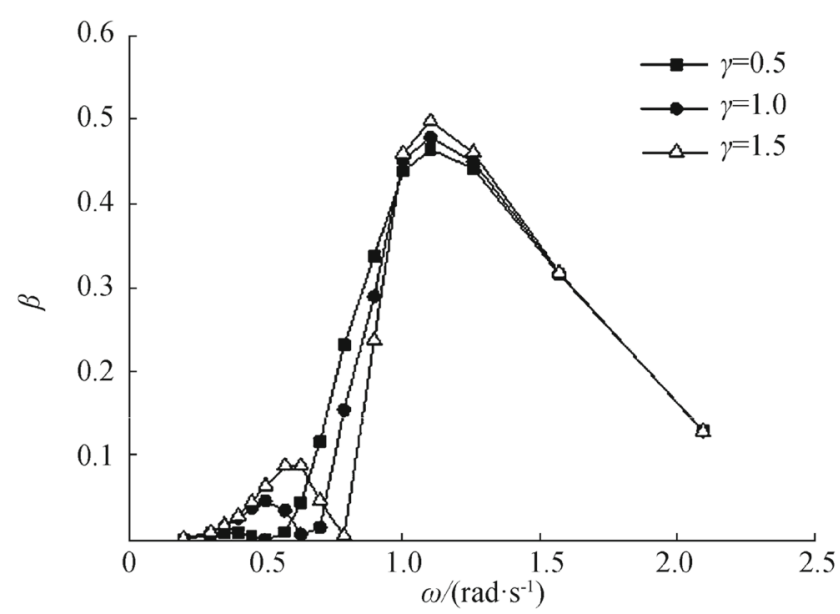

(b) Absorption powers

Fig. 6 Capture width ratios and absorption powers for different values of $B_{\text {pto }}$ and $\omega$ 
resonance occurs and the peaks of the absorption power and capture width ratio occur when the relative motion between the floats is large; when $\varepsilon=1$ or 2 , a large $K_{\text {pto }}$ leads to a large resonant frequency of the lower float, and sharp peaks in the absorption power and corresponding displacements in the two floats occur. The lower float is resonant in the low-frequency regime, which leads to sharp peaks in the absorption power and corresponding upperand lower-float displacements. The absorption power curves in Fig. 5a and the capture width ratio in Fig. 5b exhibit similar trends. When $\varepsilon=1$, the conversion efficiency in the low-frequency regime is greater than that at $\varepsilon=0$, but the peak of the conversion efficiency still occurs in the high-frequency regime. This is because the conversion efficiency is related to the incident wave power in addition to the absorption power.

At high frequency with $\omega>0.75$, the absorption power and capture width ratio decrease with increases in $\varepsilon$ (Fig. 5a and b). The relative displacements of the two floats decrease with increases in $\varepsilon$ (Fig. $5 \mathrm{c}, \mathrm{d}, \mathrm{e}$ ). This is because as the PTO stiffness increases, the motions of the two floats are similar, which leads to decreases in the absorption power.

\subsection{Effect of $K_{\mathrm{m}}$ on Response and Conversion Efficiency}

In this section, we analyze the effect $K_{m}$ on the conversion efficiency of the WEC. If we define $\gamma=K_{m} /\left(4 \times 10^{5} \mathrm{~N} / \mathrm{m}\right)$, when $B_{\text {pto }}=4 \times 10^{5} \mathrm{~N} \cdot \mathrm{s} / \mathrm{m}$ and $K_{\text {pto }}=2 \times 10^{4} \mathrm{~N} / \mathrm{m}$, and let $\gamma=0.5,1$, and 1.5, in Fig. $6 \mathrm{a}$ and b, it shows the corresponding absorption powers and conversion efficiencies of the WEC. Both the absorption power curve and conversion efficiency curve have two peak values. The first peak value increases as $\gamma$ increases and the corresponding peak frequency becomes slightly higher. The second peak value increases slightly as $\gamma$ increases and the corresponding peak frequency does not change. This is because in Eq. (47), an increase in $K_{m}$ directly leads to an increase in the natural frequency of the lower float. Therefore, the first peak frequency becomes higher but the second peak frequency does not change as $\gamma$ increases. An increase in the $K_{m}$ value leads to an increase in the constraint force exerted on the lower float, which prevents the lower float from moving with the upper float. Therefore, the relative motion between the two floats increases and both peak values increase as $\gamma$ increases. According to Fig. 5a and c, when $K_{\text {pto }}$ is small, the peak value appearing at $\omega=1.1 \mathrm{rad} / \mathrm{s}$ is generated by the large relative motion between the two floats, whereas the first peak value is generated by the resonance of the lower float. Therefore, as $\gamma$ increases, the increase in the first peak value is much larger than that of the second peak value. When designing WECs, $K_{m}$ can be made large to increase the natural frequency of the lower float and thus guarantee a greater absorption power over a wider spectrum.

\section{Conclusions}

(1) When the PTO damping coefficient is at its optimal value $B_{\mathrm{opt}}$, there is a relationship between the conversion efficiency of the WEC and the natural frequencies of the two floats. Two peak values occur when the two floats are resonant. When the lower float is resonant, the displacements of both floats are too large for the purposes of engineering. On the other hand, when the upper float is resonant, the displacements of both floats are within $1 \mathrm{~m}$. Therefore, when designing a WEC, it is better to make the upper float resonant or increase the mooring stiffness of the lower float to confine its resonant motion.

(2) The PTO stiffness coefficient $K_{\text {pto }}$ greatly influences the natural frequency of the lower float. As $K_{\text {pto }}$ increases, the natural frequency of the lower float increases and the peak value of the absorption power moves to a lowfrequency regime. In a high-frequency regime, as $K_{\text {pto }}$ increases, the conversion efficiency decreases. Thus, a proper $K_{\text {pto }}$ is needed to confine the conversion efficiency to a low-frequency regime and to avoid reducing a high-frequency regime too much.

(3) The mooring stiffness coefficient $K_{m}$ has a direct influence on the natural frequency of the lower float but has no apparent influence on the upper float. When designing a WEC, if the geometric parameters of the upper and lower floats are defined, $K_{m}$ can be used to adjust the natural frequency of the lower float.

Funding Information This work was supported by the National Natural Science Foundation of China (51409066, 51761135013), High Technology Ship Scientific Research Project from the Ministry of Industry and Information Technology of the People's Republic of China-Floating Security Platform Project (the second stage, 201622), and the Fundamental Research Fund for the Central University (HEUCFJ180104, HEUCFP1809).

Open Access This article is distributed under the terms of the Creative Commons Attribution 4.0 International License (http:// creativecommons.org/licenses/by/4.0/), which permits unrestricted use, distribution, and reproduction in any medium, provided you give appropriate credit to the original author(s) and the source, provide a link to the Creative Commons license, and indicate if changes were made.

\section{References}

Basco RD (1984) Water wave mechanics for engineers and scientists. Prentice-Hall, New Jersey, 245-255. https://doi.org/10.1142/1232

Cho IH, Kim MH (2017) Hydrodynamic performance evaluation of a wave energy converter with two concentric vertical cylinders by analytic solutions and model tests. Ocean Eng 130:498-509. https://doi.org/10.1016/j.oceaneng.2016.11.069

Dai Y, Chen Y, Xie L (2017) A study on a novel two-body floating wave energy converter. Ocean Eng 130:407-416. https://doi.org/10.1016/ j.oceaneng.2016.11.049 
Eriksson M, Isberg J, Leijon M (2005) Hydrodynamic modelling of a direct drive wave energy converter. Int J Eng Sci 43(17-18):13771387. https://doi.org/10.1016/j.ijengsci.2005.05.014

Evans DV (1976) A theory for wave-power absorption by oscillating bodies. J Fluid Mech 77(1):1-25. https://doi.org/10.1017/ S0022112076001109

Falcao A (2010) Wave energy utilization: a review of the technologies. Renew Sust Energ Rev 14:899-918. https://doi.org/10.1016/j.rser. 2009.11.003

Falnes J (1999) Wave-energy conversion through relative motion between two single-mode oscillating bodies. J Offshore Arct Eng 121(1):32-38. https://doi.org/10.1115/1.2829552

Falnes J (2007) A review of wave-energy extraction. Mar Struct 20(4): 185-201. https://doi.org/10.1016/j.marstruc.2007.09.001

Isaacs JD, Seymour RJ (1973) The ocean as a power resource. Int J Environ Stud 4(3):201-205. https://doi.org/10.1080/ 00207237308709563

Kim J, Koh HJ, Cho IH, Kim MH, Kweon HM (2016) Experimental study of wave energy extraction by a dual-buoy heaving system. Int J Naval Archit Ocean Eng 9(1):25-34. https://doi.org/10.1016/ j.ijnaoe.2016.07.002

Liang C, Zuo L (2017) On the dynamics and design of a two-body wave energy converter. Renew Energy 101(1):265-274. https://doi.org/ 10.1016/j.renene.2016.08.059

Son D, Yeung RW (2017) Optimizing Ocean-wave energy extraction of a dual coaxial-cylinder WEC using nonlinear model predictive control. Appl Energy 187:746-757. https://doi.org/10.1016/j.apenergy. 2016.11.068
Son D, Belissen V, Yeung RW (2016) Performance validation and optimization of a dual coaxial-cylinder ocean-wave energy extractor. Renew Energy 92:192-201. https://doi.org/10.1016/j.renene.2016.01.032

Tanizawa K (1995) A nonlinear simulation method of 3-D body motions in waves. J Soc Naval Architect 178:179-191. https://doi.org/10. 2534/jjasnaoe1968.1995.178_179

Wu BJ, Wang X, Diao XH, Zhang YQ (2013) Response and conversion efficiency of a wave energy device consisting of double cylindrical floats with two degrees of freedom. Science China Physics, Mechanics Astronomy 43(8):978-986. https://doi.org/10.1360/ $132012-598$

Yu YH, Li Y (2013) Reynolds-averaged Navier Stokes simulation of the heave performance of a two-body floating-point absorber wave energy system. Comput Fluids 73(6):104-114. https://doi.org/10. 1016/j.compfluid.2012.10.007

Zhang WC, Liu HX, Zhang L, Zhang XW (2016) Hydrodynamic analysis and shape optimization for vertical axisymmetric wave energy converters. China Ocean Eng 30(6):954-966. https://doi.org/10.1007/ s13344-016-0062-2

Zhou BZ, Wu GX (2015) Resonance of a tension leg platform exited by third-harmonic force in nonlinear regular waves. Philos Trans R Soc A Math Phys Eng Sci 373(2033):156-164. https://doi.org/10.1098/ rsta.2014.0105

Zhou BZ, Wu GX, Teng B (2015) Fully nonlinear wave interaction with freely floating non-wall-sided structures. Eng Anal Bound Elem 50: 117-132. https://doi.org/10.1016/j.enganabound.2014.08.003

Zhou BZ, Wu GX, Meng Q (2016) Interactions of fully nonlinear solitary wave with a freely floating vertical cylinder. Eng Anal Bound Elem 69:119-131. https://doi.org/10.1016/j.enganabound.2016.05.004 